\title{
Cancer Survivorship in General Practice - Barriers Encountered by General Practitioners in Ireland
}

\author{
Marie O'Shea ${ }^{1}$, Claire Collins, ${ }^{2, *}$ \\ ${ }^{1}$ Research Officer, Irish College of General Practitioners, Ireland \\ ${ }^{2}$ Director of Research and Innovation, Irish College of General Practitioners, Ireland
}

Copyright $@ 2018$ by authors, all rights reserved. Authors agree that this article remains permanently open access under the terms of the Creative Commons Attribution License 4.0 International License

\begin{abstract}
Cancer survivors may experience a wide range of complex health issues as a result of their cancer type and treatment. With increased rates of health care utilisation compared to non-cancer patients, the transition back into the primary care setting post-cancer treatment can be difficult for both GPs and patients to navigate. To identify the current barriers encountered by GPs in Ireland in the area of cancer survivorship, an online survey of GPs was undertaken. A total of 514 completed surveys were analysed, a response rate of $18.2 \%$, which is typical of response rates from GPs in Ireland and internationally. The majority of GPs had never attended a survivorship course, conference or workshop. Only $17.2 \%$ and $19.7 \%$ of GPs respectively considered that they had enough information on the possible long-term issues from cancer treatments. Further education on cancer survivorship and improved access to services for GPs is necessary in order for the healthcare system in Ireland to be prepared to provide improved cancer survivorship care.
\end{abstract}

Keywords Cancer, Survivorship, General Practice, Family Medicine, Education

\section{Introduction}

There are an increasing number of cancer survivors living five or more years post diagnosis. Improvements in screening, early detection, diagnosis and treatment have contributed to an increase in the number of patients living with and or beyond cancer in recent decades [1-4]. Over the next two decades, cancer incidence and prevalence rates are expected to increase worldwide by approximately 65\% [5]. By 2044 in the Republic of Ireland, cancer incidence is expected to increase two-fold [6]. In Ireland, survival estimates for many cancers including colorectal, breast, kidney, testicular and prostate cancers and for multiple myeloma, lymphoma and leukaemia have improved gradually, however this trend is not applicable to all cancers. Ireland is approximately mid-way in the ranking of survival estimates in Europe for ten specific cancer types [2]. There is a lack of consistent definition in the literature in relation to cancer survivorship. However, there is some consensus that cancer survivorship relates to patients who live beyond the initial phase of cancer treatment and are cured, in remission or living with cancer $[7,8]$. Patients who survive the initial treatment period may require a range of additional medical care including treatment to manage side effects and for co-morbidities linked with their cancer type/treatment, psycho-social support and a range of medical counselling. They will also likely be tied into a series of surveillance and screening schedules to reduce risk of recurrence following the diagnosis and treatment phases [9]. Cancer survivors have increased rates of health care consumption at a primary care level [9-12].

Providing care to cancer survivors in general practice can be particularly challenging due to the complexities of these patients healthcare needs, a lack of access to appropriate services, unclear care pathways, late effects of the disease, insufficient access to information with secondary care providers, and lack of appropriate education and training $[13,14]$. International research has identified that at a primary care level, practitioners lack knowledge and necessary education on the impact of cancer treatments and the long terms effects of living with cancer [15-17]. In Ireland, there is a dearth of literature on the impact of cancer survivorship on general practice. Currently there are no clear recommendations, evidence-based guidelines, or educational recourses to support GPs in managing the consequences of cancer. One section of an Irish based 2015 survey on GP experience with the work of the National Cancer Control Programme (NCCP) sought to assess the supports GPs require regarding cancer survivorship and additional cancer care education [18]. This paper outlines and addresses these findings. 


\section{Methods}

This research consisted of a descriptive literature review and a quantitative survey of GPs. The aim of this survey was to obtain the views and experiences of Irish general practitioners in relation to the work of the National Cancer Care Programme (NCCP), as a follow-up to the 2006 ICGP/Irish Cancer Society 'Early detection of cancer: a needs assessment of general practitioners' study [19]. The survey also sought to gain GP opinion in relation to the future priorities of the NCCP which would assist GPs in their work, and current barriers encountered by GPs and their patients with suspected or confirmed cancer. A project advisory group was created to oversee the research. In April 2015, postal questionnaires (see Appendix) were sent to 2,822 ICGP members in the Republic of Ireland, excluding retired GPs and trainees. A letter of introduction was included with the questionnaire, which outlined the purpose of the study and the data collection process. Freepost return envelopes were included to encourage response. A postal reminder was sent two weeks later. A notice encouraging response was also placed in the monthly journal of the Irish College of General Practitioners. Return of a completed anonymous questionnaire was taken as implied consent. Data analysis in the SPSS Version 22 yielded frequency distributions, descriptive statistics and cross-tabulations. Qualitative open ended comment data were manually thematically coded.

\section{Results}

The survey was posted to 2,824 GPs. A total of 514 completed surveys from individual GPs were included in the final analysis, a response rate of $18.2 \%$.

\section{Participant and Practice Profile}

In terms of the profile of respondents, $53.4 \%(n=270)$ were female and $46.6 \%$ (236) were male. Nearly $42 \%$ of respondents $(\mathrm{n}=210)$ were over 25 years in general practice, $35.3 \%(\mathrm{n}=177)$ were between $11-25$ years and $22.9 \%$ $(n=115)$ were in practice 10 years or less. All counties in the Republic of Ireland were represented, with the majority of respondent practices based in Dublin (26.6\%, $\mathrm{n}=128)$, Cork $(14.1 \%, \mathrm{n}=70)$ and Galway $(6.9 \%, \mathrm{n}=34)$. Overall, $36.4 \%$ of the respondents worked in single handed practices. Just over $85 \%(n=433)$ of GPs had a practice nurse in situ. Nearly $46 \%(n=232)$ of practices were based less than five miles from the nearest hospital to which a GP could refer a patient for assessment of suspected cancer (Table 1). Thirty five percent $(\mathrm{n}=176)$ of practices were less than 5 miles from the nearest designated cancer centre; $22.9 \%$ and $22.5 \%$, were between $5-25$ miles and 26-50 miles from the nearest centre.

Table 1. Respondent demographics

\begin{tabular}{|l|c|}
\hline \multicolumn{1}{|c|}{ Sex } & $\mathrm{n} / \%$ \\
\hline Female & $270(53.4 \%)$ \\
\hline Male Years in General Practice & $236(46.6 \%)$ \\
\hline \multicolumn{1}{|c|}{ (10yrs or $<$} & $115(22.9 \%)$ \\
\hline $11-25 y r s$ & $177(35.3 \%)$ \\
\hline$>25 y r s \quad 210(41.8 \%)$ \\
\hline \multicolumn{1}{|c|}{ Distance to nearest hospital } \\
\hline$<5$ miles & $232(45.8 \%)$ \\
\hline $5-25$ miles & $173(34.2 \%)$ \\
\hline $26-50$ miles & $83(16.4 \%)$ \\
\hline$>50$ miles & $18(3.6 \%)$ \\
\hline \multicolumn{1}{|c|}{ Distance to nearest designated cancer } & $176(35.4 \%)$ \\
\hline$<5$ miles & $112(22.5 \%)$ \\
\hline $5-25$ miles & $114(22.9 \%)$ \\
\hline $26-50$ miles & $95(19.1 \%)$ \\
\hline$>50$ miles & \\
\hline
\end{tabular}

\section{Sharing Care and Information}

Nearly $60 \%$ of respondents reported that they received enough detail immediately following diagnosis, while $53.4 \%$ of GPs reported receiving information during treatment, and $60.5 \%$ post treatment. It was found that one fifth of respondents rarely shared follow-up care for their patients with oncology consultants, and nearly $10 \%$ of respondents had never practiced sharing follow-up care in this situation. However, $10.7 \%$ of respondents reported that they always, $24.5 \%$ often and $35 \%$ sometimes share care with relevant oncology consultants.

Other than 'lifestyle modification factors' (70.1\%), 'bone health' (67.6\%) and 'cancer support groups' (53\%), respondents indicated that they did not have enough information in respect of many areas to provide care to their patients (Figure 1). Eighty three percent and $80.3 \%$ of respondents respectively considered that they did not have enough information on the 'possible long-term issues from chemotherapy' and 'possible long-term issues from radiation therapy'. Over $76 \%$ of GPs considered that they did not have sufficient information on 'recommended evidence based surveillance for patients with a previous cancer', while nearly $70 \%$ did not have enough information on factors related to living with cancer. 


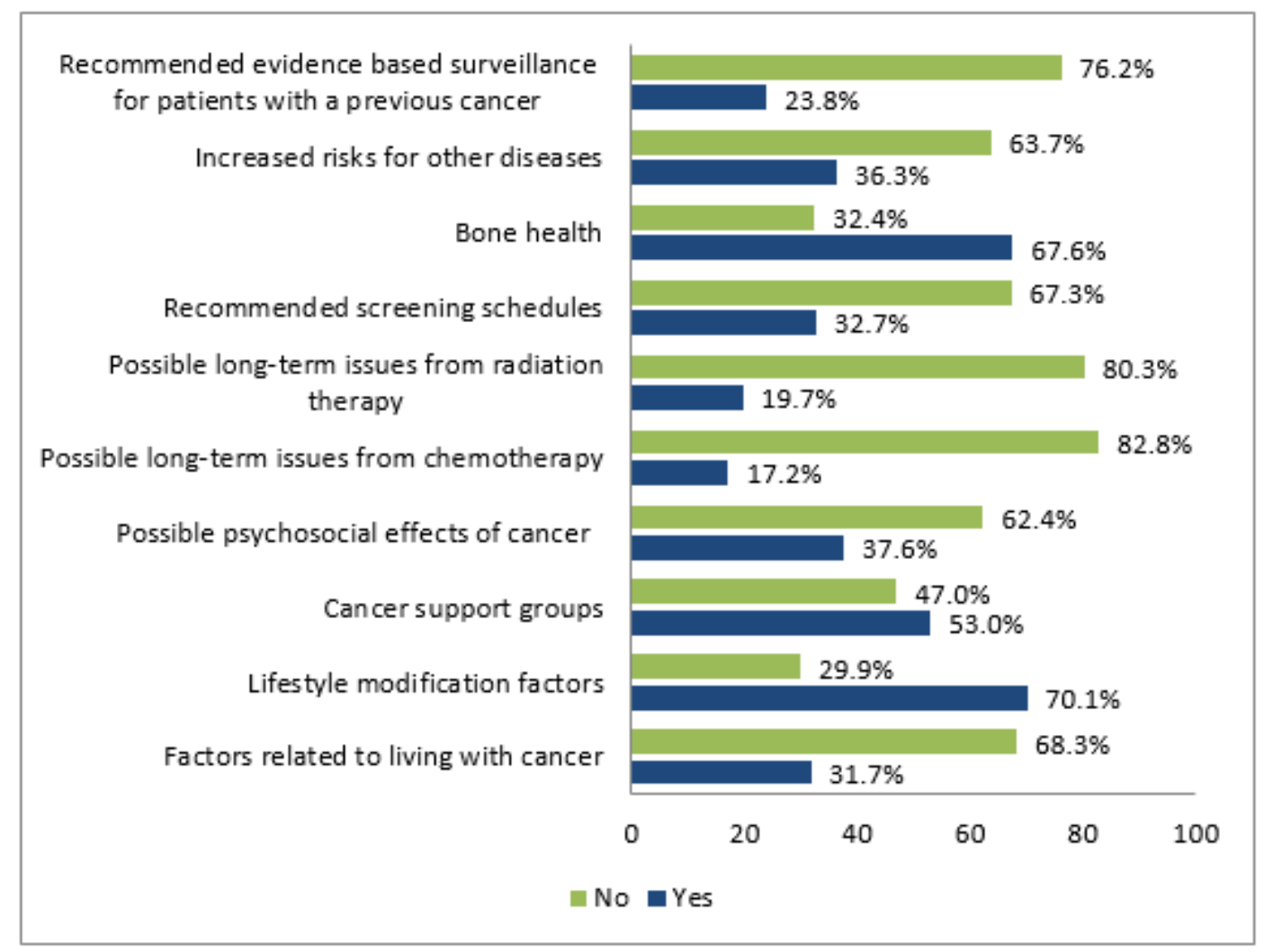

Figure 1. GP access to relevant information to provide patient care

\section{GP Education}

The vast majority of respondents (93.5\%) had never attended a course, conference or workshop on the subject area of cancer survivorship (no specific definition of 'cancer survivorship' was provided but this was left to the respondent's interpretation). Approximately $60 \%$ of respondents indicated that they would like to receive further education on this subject. Respondents were asked to indicate from a predefined list the topics they would most like to receive further education on. 'Complications of chemotherapy' (65\%), 'complications of radiation therapy' (62.3\%), 'cancer screening' (57.4\%) and 'NCCP referral guidelines' (57\%) were the most commonly selected topics (Figure 2). 'Smoking cessation' ranked the lowest at $8.6 \%$. Where 'other' was given as a response, sixteen respondents identified alternative topic areas they regarded as necessary to receive further education. These included 'symptom management', 'counselling', 'evidence based surveillance of patients with a previous cancer' and 'risk factors for families'. Three respondents commented that due to time restraints in general practice, they were unavailable to undertake further education. 'Online modules', a 'hybrid of online, face to face and manual' formats and 'evening seminars' were the preferred formats to receive further cancer specific education. Respondents were given the opportunity to comment via open ended response; twelve respondents proposed that continuous medical education (CME) meetings were the most appropriate format to receive additional education.

\section{Service Availability and Education for Patients}

In Ireland, the General Medical Scheme (GMS) enables GPs to enter into contracts with the national Health Service Executive (HSE) to provide health services and medicines to patients covered by medical cards free of charge. As of 2016, there are approximately 1.26 million patients in Ireland registered under the scheme. Respondents were asked to select from a predefined list of ten services all which were accessible to their GMS and private patients. All services, except one (smoking cessation services), were more commonly available to respondent's private patients than GMS patients (Figure 3). 'Pain and symptom management programmes', 'dietician' and 'smoking cessation' services were most commonly available to both GMS and private patients. However, the remaining services were accessible for less than $50 \%$ of private patients, and $40 \%$ of GMS patients. Nearly two-thirds of respondents reported that 'cancer rehabilitation programmes' were unavailable to their GMS (66.5\%) or private patients $(60 \%)$. Similar high percentages identifying a lack of availability were reported for 'family', 'fertility', 'sexual' and 'genetic cancer' counselling and resources. 


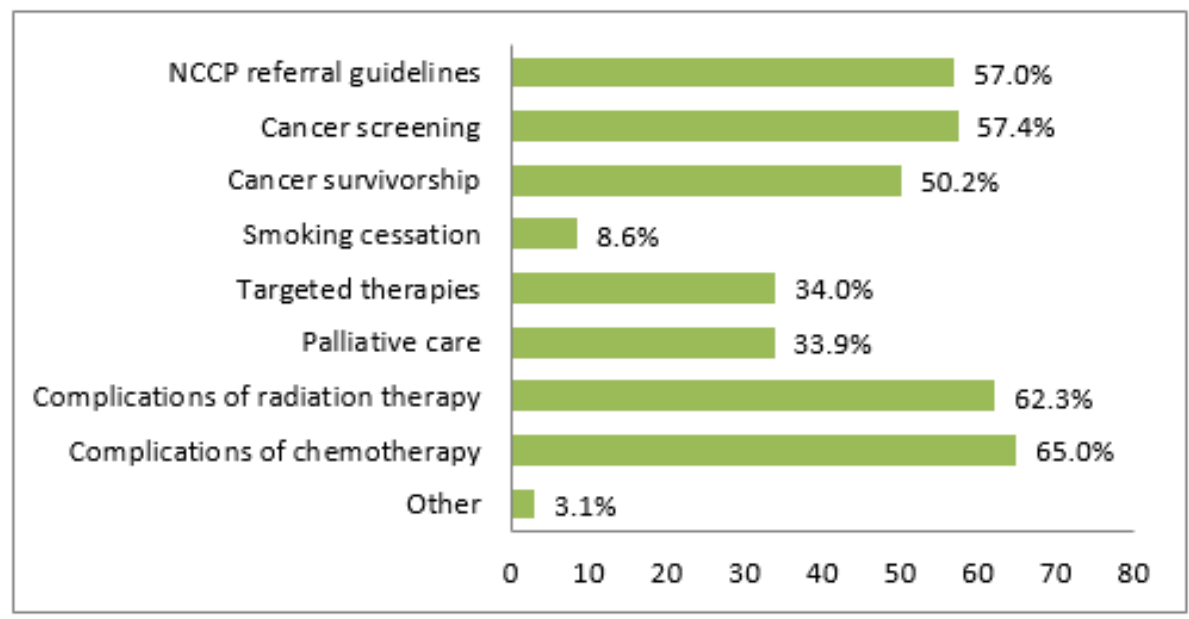

Figure 2. Areas in which GPs wish to receive further education

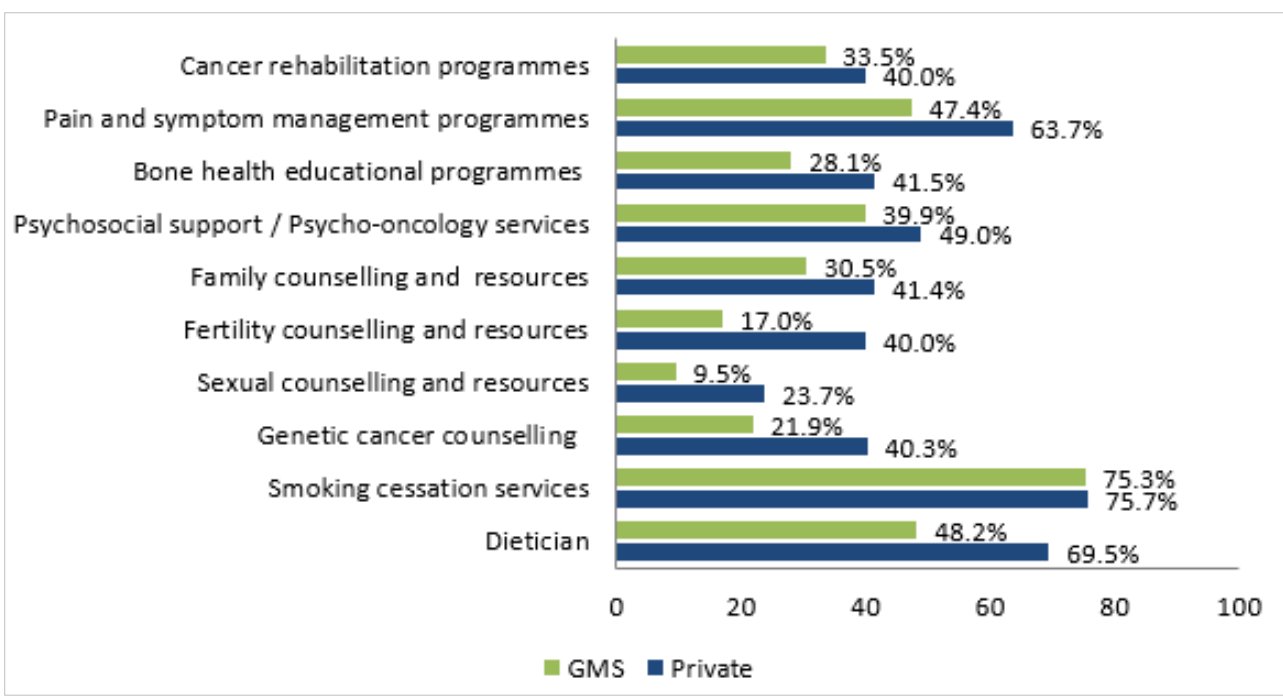

Figure 3. GPs views on whether services are readily access to their GMS and private patients

The vast majority of respondents reported that they had addressed the risks of 'smoking' (99.2\%) and 'UV exposure' (96\%) on patient health with their patients. Between $72 \%$ and $90 \%$ of respondents also highlighted the risks related to 'alcohol', 'obesity' and 'lack of exercise'.

\section{Discussion}

For the purpose of this paper, we explored GPs views and experiences in managing cancer survivorship in general practice, with the aim of highlighting any knowledge gaps and issues GPs may experience accessing necessary information and services. The profile of respondents is consistent with available ICGP statistics on the profile of GPs in Ireland. Years in general practice also corresponds with ICGP data from a previous cancer detection survey [19]. Given the evidence that people are living substantially longer with cancer or surviving cancer, major changes and challenges are emerging in general practice in the area of cancer survivorship care. This Irish study yielded important findings with regard to GP views on the supports and education they deem necessary to assist them in the care of patients affected by cancer. A large cohort of GPs would welcome further education to aid their patient centred care, particularly on the complications of cancer treatments, the possible long term effects of living post-cancer treatment and increased risks of co-morbidities. Significant gaps were identified in service availability for patients, and in the supports available to GPs for the optimum delivery of survivorship care to cancer patients in Ireland. International literature suggests that difficult or delayed communication between hospitals and GPs can prevent GPs from optimally managing patients [13, 20, 21]. A need for greater continued information sharing between GPs and secondary care teams has been recognised.

We acknowledge that undertaking further education and accessing necessary supports and services is not without its problems. A minority of GPs identified that further education was not a viable option for them at a time in which general practice is under increasing stress, particularly for GPs working in single handed practices. At 
a systems level, respondents identified evident barriers in accessing necessary healthcare, particularly for patients from lower socioeconomic groups. Health inequalities in accessing specialist services are prevalent in Ireland [18, 22]. This survey identified that psychosocial, psycho-oncological and counselling for genetic cancers, sexual and fertility issues in particular were limited for these patients which suggest the necessity for extended access to services to support the management of cancer patients in general practice.

The overall response rate is a limiting factor; however, surveys of GPs typically result in lower response rates than surveys of other medical professionals and the general population. Such low response rates are thought to be related to severe time restraints and increasing pressures in general practice [23]. Nevertheless, the response rate is consistent with other international and Irish GP surveys [18, 24, 25]. Additionally, the profile of those respondents who did reply is consistent with the national GP population and is therefore generalisable. As the survey participants were informed about the focus of the survey via the letter of introduction and in a news piece in the FORUM journal, it may be likely that respondents were those more interested in offering feedback to the NCCP, than the general GP population.

By 2040, the number of new invasive cancer cases is estimated to increase. As such, the healthcare system in Ireland, and medical education and training must adapt and prepare for improved cancer survivorship care.

Key recommendations:
A large cohort of GPs would welcome further education to aid
their patient centred care, particularly on the complications of
cancer treatments, the possible long term effects of living
post-cancer treatment and increased risks of co-morbidities.
Significant gaps exist in the supports available to GPs for the
optimum delivery of survivorship care to cancer patients in
Ireland.
A need for greater continued information sharing between
GPs and secondary care teams has been recognized in the
literature.
Extended access to services (such as psychosocial,
psycho-oncological and counselling for genetic cancers,
sexual and fertility counselling) to support the management of
cancer patients in general practice is required.

\section{Compliance with Ethical Standards}

Funding for this project was provided by the National Cancer Control Programme (NCCP).

The authors nor the authors' institution has financial or personal relationships with other persons or organisations that inappropriately influence (bias) their actions.

The authors would like to express her thanks to all who participated in the study, with a particular mention to the GPs who responded to the survey.

\section{Conflict of Interest}

The authors have not conflict of interest.

Ethical approval: All procedures performed in studies involving human participants were in accordance with the ethical standards of the institutional and/or national research committee and with the 1964 Helsinki declaration and its later amendments or comparable ethical standards.

Informed consent: Respondents were supplied with full information regarding this study. An anonymous questionnaire online was returned. As such no separate written consent was obtained but consent was implied by completion of the anonymous online survey questionnaire.

\section{REFERENCES}

[1] Richards, M.A. The size of the prize for earlier diagnosis of cancer in England. Br J Cancer. 2009; 3(101):125-9.

[2] Banks, J., Hollinghurst, S., Bigwood, L., Peters, T.J., Walter, F.M. and Hamilton, W. Preferences for cancer investigation: a vignette-based study of primary-care attendees. Lancet Oncol. 2014; 15:232-40.

[3] Redaniel, M.T., Martin, R.M., Ridd, M.J., Wade, J. and Jeffreys, M. Diagnostic Intervals and Its Association with Breast, Prostate, Lung and Colorectal Cancer Survival in England: Historical Cohort Study Using the Clinical Practice Research Datalink, PLoS ONE. 2015; 10(5).

[4] Baili, P., Di Salvo, F., Marcos-Gragera, R., Siesling, S. Mallone, S., Santaquilani, M., Lillini, R., Francisci, S. and and the EUROCARE-5 Working Group. Age and case mix-standardised survival for all cancer patients in Europe 1999-2007: Results of EUROCARE-5, a population-based study. European Journal of Cancer. 2015; 51(15): 2120-2129.

[5] Stewart BW, Wild CP, editors. World Cancer Report 2014. International Agency for Research on Cancer: Lyon, France; 2014.

[6] National Cancer Registry. Cancer projections for Ireland 2015 - 2040. National Cancer Registry: Cork, Ireland; 2014.

[7] Hoekstra, R.A., Heins, M. J. and Korevaar, J.C. Health care needs of cancer survivors in general practice: a systematic review. BMC Family Practice. 2014; 15:94.

[8] Marzorati, C., Riva, S. and Pravettoni, G. Who Is a Cancer Survivor? A Systematic Review of Published Definitions. Journal of Cancer Education. 2016; 1-10.

[9] Heins, M., Korevaar, J., Rijken, P. and Schellevis, F. For which health problems do cancer survivors visit their general practitioner? Eur J Canc. 2013; 49(1):211-8.

[10] Khan, N.F., Watson, E. and Rose, P.W. Primary care consultation behaviours of long-term, adult survivors of cancer in the UK. Br J Gen Pract. 2011; 61(584):197-199.

[11] Christensen, K.G., Fenger-Grøn, M., Flarup, K.R. and Vedsted, P. Use of general practice, diagnostic investigations and hospital services before and after cancer 
diagnosis - a population-based nationwide registry study of 127,000 incident adult cancer patients. BMC Health Serv Res. 2012; 12:224.

[12] Lawrence, R.A., McLoone, J.K., Wakefield C.E. and Cohn, R.A. Primary Care Physicians' Perspectives of Their Role in Cancer Care: A Systematic Review. Journal of General Internal Medicine. 2015; 1-15.

[13] Farquhar, M.C., Barclay, S.I., Earl, H., Grande, G.E., Emery, J. and Crawford, R.A. Barriers to effective communication across the primary/secondary interface: examples from the ovarian cancer patient journey (a qualitative study). Eur J Cancer Care. 2005; 14:359-66.

[14] Guassora, A.D., Jarlbaek, L. and Thorsen, T. Preparing general practitioners to receive cancer patients following treatment in secondary care: a qualitative study. BMC Health Services Research. 2015; 15:202.

[15] Nissen, M.J., Beran, M.S., Lee, M.W., Mehta, S.R., Pine, D.A. and Swenson, K.K. Views of primary care providers on follow-up care of cancer patients. Family Medicine. 2007; 39:477-82.

[16] Fitch, M.I. Looking to the Future: Cancer Survivorship in Canada. Canadian Partnership against Cancer [Internet]. 2012 (cited 2016 August 2). Available from: http://www.caot.ca/pdfs/PIF/P2\%20PIF.pdf

[17] Luctkar-Flude, M., Aiken, A., McColl, M.A., Tranmer, J. A comprehensive framework and key guideline recommendations for the provision of evidence-based breast cancer survivorship care within the primary care setting. Family Practice. 2015; 32(2):129-140.
[18] O’Shea, M.T. and Collins, C. A survey of GP experience with the work of the National Cancer Control Programme and their views in relation to service priorities. Irish College of General Practitioners: Dublin, Ireland; 2016.

[19] Daly, H. and Collins, C. Barriers to Early Diagnosis of Cancer in Primary Care: A Needs Assessment of GPs. Ir Med J. 2007; 100(10):624-6.

[20] Harris, M.F. and Harris, E. Facing the challenges: general practice in 2020. Medical Journal of Australia. 2006; 185:122-124.

[21] Rowlands, S., Callen, J. and Westbrook, J. What information do general practitioners need to care for patients with lung cancer? A survey of general practitioners' perceptions. Health Information Management Journal. 2010; 39:8-15.

[22] O'Riordan, M., Collins, C, Doran, G. Access to Diagnostics a key enabler for a primary care led health service. Irish College of General Practitioners: Dublin, Ireland; 2013.

[23] O’Brien, J.G., Long, V., Collins, C., Ni Rian, A. and O’Neill, D. Elder Abuse and neglect: A Survey of Irish General Practitioners. Journal of Elder Abuse and Neglect. 2013; 26(3).

[24] Byrne, M., Doherty, S., McGee, H. and Murphy, A.W. General practitioner views about discussing sexual issues with patients with coronary heart disease: a national survey in Ireland. BMC Family Practice. 2010; 10:11-40.

[25] Van Geest, J.B., Johnson, T.P. and Welch, V.L. Methodologies for Improving Response Rates in Surveys of Physicians: A Systematic Review. Evaluation in the Health Professions. 2007; 30(4):303-21. 